\title{
Extrait du procès verbal de l'académie impériale des sciences de St. Pétersbourg du 24 Septembre (6 Octobre) ${ }^{\star}$ ).
}

M. Fuss lit une note conçue en ces termes: „Lorsque, il y a seize ans, je fus chargé du secrétariat de l'académie, un de mes premiers soins fut l'inspection de nos archives. J'y trouvai, entre autres, quelques paquets de la correspondance de notre immortel Euler, en date pour la plupart, des années quarantièmes et cinquantièmes du siècle dernier, c'est à dire du tems de son service en Prusse; puis quelques lettres des 14 années antérieures à cette époque, et où il appartenait encore à la Russie, mais rien, ou presque rien des vingt dernières années de sa vie qu'il passa de nouveau au sein de notre Académie. Ces lettres étaient rangées par ordre chronologique et formaient une dixaine de paquets isolés. J'y trouvai, comme je devais m'y attendre, au milieu d'une foule de noms obscurs, quelques noms illustres qui, de nos jours encore, brillent d'un éclat impérissable dans les annales des sciences, - au milieu de lettres remplies des phrases banales de l'adulation, d'affaires de service d'un intérêt passager, ou d'objets qui, alors même, n'offraient de l'intérêt qu'aux auteurs de ces lettres, je trouvai, dis-je, dans toute cette ivraie, un nombre assez considérable de grains précieux qui, aujourd'hui encore, méritent d'être conservés et offerts aux géomètres. Je n'ai qu'à vous citer 10 lettres de Jean Bernoulli l'aîné, illustre coïnventeur du calcul infinitésimal, l'ami de Leibnitz et le maître de notre Euler; 63 lettres de Daniel Bernoulli, fils et rival redouté du précédent; 4 lettres de Nicolas Bcrnoulli, cousingermain de Daniel, auteur de l'Ars conjectandi in jure et qui, avec Montmort, culti.va avec tant de succès l'analyse des probabilités dont son oncle Jacques avait jeté les premiers fondemens, -6 lettres de Gabriel Cramer de Genève, auteur de l'analyse des lignes courbes algébriques etc. etc.

Ce furent d'abord les lettres des Bernoulli qui attirèrent mon attention particulière. Je fus assez heureux pour pouvoir en compléter encore la suite, ayant trouvé, dans les papiers de mon père, les copies, faites de sa main, de quatre lettres de Jean Bernoulli et la traduction française d'une lettre de Daniel, qui toutes manquent à notre collection, et dont les originaux avaient vraisemblablement été retirés avant même qu'elle fût déposée aux archives, par la famille Euler. Il en est de même de deux lettres de Clairaut, d'une de Naudé et d'une de Poléni, dont je possède également des copies de la main de mon père.

Toutes ces lettres roulent sur des objets de science; celles des Bernoulli surtout offrent un haut intérêt non seulement pour l'histoire de la science et l'histoire littéraire en général, mais encore sous le rapport des méthodes et des aperçus, du raisonnement et des artifices de calcul que nul géomètre ne verra sans admiration, ni sans

*) Cet extrait a été communiqué à l'éditeur de ce jonrnal par M. Fuss, sécretaire perp. de l'acadénie, avec permission de l'insérer dans le journal des Math. 
y puiser quelque instruction. Quant à moi, la jouissance que m'a procurée l'étude de ces lettres n'a été troublée que par le regret, que j'ai épprouvé à chaque page, de ne pas ponvoir lire en mème temps les réponses d'Euler. A coup sûr, celles-ci eussent décuplé la valeur de cette précieuse collection. Malheureusement tous mes efforts pour me les procurer ont été infructueux (je me suis mis en rapport à cet effet avec l'université de Bâle et avec M. le professeur Bernoulli de cette ville, descendant en ligne droite de Jean et de Daniel). Néanmoins j'ai la conviction que la publication immédiate d'un choix des lettres que nous possédons, sera accueillie avec enthonsiasme par tous les géomètres; tel, du moins, a été l'avis de nos collègues de la section mathématique que j'ai consultés à cet égard.

Les lettres des trois Bernoulli, avec celles de Cramer, de Lambert et de Clairaut formeront à elles seules un volume de 16 à 20 feuilles environ. - Nos archives renferment en outre tout un volume de lettres de Goldbach. Bien que ce géomètre ait joui de son vivant d'une grande rèputation, et qu'Euler lui-mème, ainsi qu'on le voit par un passage remarquable des lettres de Daniel, eût beaucoup d'estime et d'amitié pour lui; cependant, l'oubli, dans lequel est tombé son nom, et l'intérèt secondaire qu'offrent ses lettres, - quoique toutes savantes, - m'avait déterminé à ne pas les comprendre dans le recueil que je méditais. Or, je viens d'apprendre qu'il existe aux archives centrales de Moscou plusieurs paquets renfermant les réponses d'Euler à Goldbach. Cette circonstance change entièrement la face de la question: les réponses d'Euler donneront aux lettres de Goldbach un degré d'importance que, prises isolément, elles n'avaient pas, et la publication de la correspondance complète de ces deux savans offrira, sans aucun doute, des données fort intéressantes pour l'histoire des mathémathiques en général et pour celle des travaux d'Euler en particulier. J'ai l'espoir bien fondé d'obtenir de Moscou soit les lettres originales d'Euler, soit la permission d'en faire tirer copie. *)

Depuis que les sciences ont cessé d'ètre la propriété exclusive d'un petit nombre d'initiés, ce commerce épistulaire des savans a été absorbé par la presse périodique. Le progrés est incontestable. Cependant, cet abandon avec lequel on se communiquait autrefois ses idées et ses découvertes, dans des lettres toutes confidentielles et privées, - on ne le retrouve plus dans les pièces mûries et imprimées. Alors, la vie du savant se reflétait, pour ainsi dire, tout entière dans cette correspondance. On y voit les grandes découvertes se préparer et se développer graduellement; pas un chainon, pas une transition n'y manque; on suit pas à pas la marche qui a conduit à ces découvertes et l'on puise de l'instruction jusque dans les erreurs des grands génies qui

*) Je me félicite de pouvoir ajouter ici, que, grâce à la libéralité éclairée de M. le Prince Obolensky, dirigeant les archives de Moscou, je me trouve, dans ce moment, dépositaire de cent lettres d'Euler à Goldbach, toutes pleines de recherches importantes sur différents snjets de la science, et particulièrement sur la théorie des nombres. La lecture de cette correspondance me fait encore plus vivement regretter la perte des lettres d'Euler aux Bernoulli. Si, par un heureux hasard, elle se retrouvaient quelque part, soit dans une collection publiqne, soit entre des mains privées, que cette annonce puisse servir aux personnes qui en seraient dépositaires, ou qui senlement en auraient connaissance, d'invitation à m'en donner avis! Ce 2 (14) novembre 1841.

Fuss. 
en furent les auteurs. Cela explique suffisamment lintérêt qui se rattache à ces sortes de correspondances, et me fait espérer que l'Académie voudra bien m'autoriser à livrer à l'impression un Choix de lettres inédites de quelques célèbres Géomètres du 18ème siècle à Léonard Euler. - On sait qu'une entreprise tout à fait analogue et relative aux écrits et à la correspondance de Leibnitz, se prépare, dans ce moment, en Allemagne.

Si l'académie veut bien entrer dans mes vues, je me permettrai d'appeler encore son attention sur un autre projet qui m'a été suggéré par M. Jacobi de Königsberg, et qui se lie fort intimément à celui que je viens de lui soumettre. M. Jacobi m'engage à publier de nouveau la liste des écrits d'Euler, fournie par mon père à la suite de son Eloge, et d'y indiquer les volumes de nos Mémoires, où sont insérées les 183 dissertations posthumes de ce grand géomètre, marquées dans l'Eloge comme inédites. Ce désir de notre savant collègue m'a rappelé un travail qu'en 1817 et 1818, j’avais exécuté pour mon propre usage, savoir un catalogue systématique de tous les écrits d'Euler, avec renvoi aux recueils académiques qui les renferment. J'ajouterai pour ceux de mes collègues qui ne sont pas mathématiciens, que le nombre de ces écrits, non compris les grands ouvrages publiés séparément, monte à plus de 700, et qu'aujourd'hui encore, 60 ans après la mort de ce grand homme, nul géomètre ne peut se dispenser de recourir souvent à ses travaux. Or, une pareille liste systématique et chronologique doit nécessairement faciliter beaucoup la recherche des pièces dont on se trouve avoir besoin. J'ai donc soumis mon travail à une nouvelle révision, et après l'avoir complété, j'ai le projet de le placer en tête du recueil épistolaire ci-dessus mentionné et d'y joindre une notice sur les écrits d'Euler. Car j'ai trouvé que certains mémoires, marqués comme inédits dans la liste, donnée par mon père dans son Eloge d'Euler, ont vraisemblablement, plus tard, été retirés de nos archives, car ils ne s'y retrouvent plus; d'autres, publiés depuis, manquent dans la liste. Il y en a d'autres encore qui, à dessein, noont pas été publiés, soit parcequ'ils sont apostillés de la main de mon père "à supprimer", soit parceque leur origine a paru douteuse. Enfin, en examinant les manuscrits d'Euler que renferment nos archives et ceux que je possède moi-même, mon catalogue systématique doit me fournir un moyen facile de déterminer au juste lesquels de ces manuscrits n'ont jamais été publiés, car la liste des mémoires inédits d'Euler, fournie par mon père, ne pouvait se rapporter qu'à ceux qui avaient été présentés à l'Académie de son vivant. C'est ainsi, p. ex., qu'une inspection toute superficielle me permet déjà de désigner positivement comme inédit un fragment volumineux, mais mis au net par Euler même, sous le titre d'Astronomia mechanica. Je ne doute pas qu'il ne s'en trouve d'autres encore, et alors il s'agira de savoir si tous ses travaux, ou seulement quelques uns d'entre eux se prêtent à la publication, question que l'Academie voudra bien soumettre à la décision d'une commission. MM. Struve, Ostrogradsky et Bouniakovsliy ont bien voulu me promettre d'avance leur assistance éclairée."

liAcadémie approuve ce projet et autorise M. Fuss à le mettre à exécution. 